\title{
A Holling Type II Pest and Natural Enemy Model with Density Dependent IPM Strategy
}

\author{
Xia Wang, Yuan Tian, and Sanyi Tang \\ School of Mathematics and Information Science, Shaanxi Normal University, Xi'an 710062, China \\ Correspondence should be addressed to Sanyi Tang; sytang@snnu.edu.cn
}

Received 15 January 2017; Revised 25 April 2017; Accepted 14 May 2017; Published 14 June 2017

Academic Editor: Andrzej Swierniak

Copyright (C) 2017 Xia Wang et al. This is an open access article distributed under the Creative Commons Attribution License, which permits unrestricted use, distribution, and reproduction in any medium, provided the original work is properly cited.

\begin{abstract}
Resource limitations and density dependent releasing of natural enemies during the pest control and integrated pest management will undoubtedly result in nonlinear impulsive control. In order to investigate the effects of those nonlinear control strategies on the successful pest control, we have proposed a pest-natural enemy system concerning integrated pest management with density dependent instant killing rate and releasing rate. In particular, the releasing rate depicts how the number of natural enemy populations released was guided by their current density at the fixed moment. The threshold condition which ensures the existence and global stability of pest-free periodic solution has been discussed first, and the effects of key parameters on the threshold condition reveal that reducing the pulse period does not always benefit pest control; that is, frequent releasing of natural enemies may not be beneficial to the eradication of pests when the density dependent releasing method has been implemented. Moreover, the forward and backward bifurcations could occur once the pest-free periodic solution becomes unstable, and the system could exist with very complex dynamics. All those results confirm that the control actions should be carefully designed once the nonlinear impulsive control measures have been taken for pest management.
\end{abstract}

\section{Introduction}

Integrated pest management (IPM), also known as integrated pest control (IPC), is a wide approach that integrates multiple control measures for economic control of pests. IPM aims to maintain the pest population below the economic injury level (EIL) by taking biological, chemical, and physical control tactics. During the pest control, regular monitor is critically important which provides some cues on how and when to apply IPM strategies [1-5].

Note that the outbreaks of pest often cause serious ecological problems and bring large economic losses. The traditional methods to reduce the harm to agriculture due to insect pests include chemical pesticides and biological control measures [6-8]. Chemical pesticide is a measure directly acting on the pest. Biological control measure releasing natural enemies is a measure to increase the number of predators to control the population of the pest. There are several different releasing methods including inoculative release and inundative release which are different tactics of biological control that affect the target pest in different ways. Augmentative control includes the periodic introduction of natural enemies [3,9]. With inundative release, predators are collected, mass-reared, and periodically released in large numbers into the pest area, which can result in an immediate reduction in the pest populations.

However, pesticide pollution has been recognized as a major health hazard to human, it can seriously change the ecological system, and it may cause damage to nontarget organisms. So, to combine these control measures together and to maximize control effects at minimum levels of control resources, a number of impulsive differential equations have been developed to investigate the dynamics of pest and enemies and to analyze the optimal control schemes [3, 4, 1013].

In 2003, Liu and Chen studied the dynamics of the Holling type 2 predator-prey model by introducing releasing predators at fixed moments [14], and the model is as follows:

$$
\frac{d x(t)}{d t}=r x(t)\left[1-\frac{x(t)}{K}\right]-\frac{a x(t) y(t)}{1+\omega x(t)}
$$




$$
\begin{array}{ll}
\frac{d y(t)}{d t}=\frac{c x(t) y(t)}{1+\omega x(t)}-d y(t), & \\
& t \neq n T, \\
x\left(t^{+}\right)=x(t), & \\
y\left(t^{+}\right)=y(t)+\sigma, & t=n T,
\end{array}
$$

where $x$ and $y$ are densities of the prey and predator populations at time $t$, respectively; $r$ denotes the intrinsic growth rate of prey; $K$ represents the environmental capacity of prey; $d$ is the death rate of the predator; $a x(t) /(1+\omega x(t))$ is the Holling type 2 functional response; $c / a$ is the rate of conversing prey into predator; $T$ is the pulse period; $\sigma$ is the release amount of predator at time points $t=n T$. From then on, the predator-prey model considering both pulse spraying pesticides and releasing natural enemies have been extensively studied by many researchers [3, 4, 13, 15-21]. Note that for all those studies the pulse functions introduced to the models could be summarized as the following generalized linear functions:

$$
\begin{aligned}
& x\left(t^{+}\right)=\left(1-p_{1}\right) x(t), \\
& y\left(t^{+}\right)=\left(1-p_{2}\right) y(t)+\sigma, \\
& t=n T,
\end{aligned}
$$

where $p_{1}$ and $p_{2}$ are the fraction of pests and predators which have been killed instantaneously after pesticide applications.

The key assumptions from the linear pulse functions shown in (2) are as follows: (a) the instant killing rate $p_{1}$ is a constant, which shows that no matter how large the number of pest populations is, the total pest killed linearly depends on its current density [22, 23]; (b) the adverse effect of pesticides on the natural enemies is also linear dependence on its current density; (c) the releasing number of natural enemies is a constant no matter how large the natural enemies remain in the field; that is, the density of natural enemies is not carefully monitored before IPM strategy is applied. However, the killing rate and releasing constant should strictly depend on a number of factors including current densities of both populations, resources limitation, and management purposes. For example, in reality, due to the resource limitation, the more proper and usual way of releasing of enemies may be the way that is guided by the density of the current natural enemies. Therefore, taking those factors into account, we propose the following model with density dependent pulse control actions:

$$
\begin{aligned}
& \frac{d x(t)}{d t}=r x(t)\left[1-\frac{x(t)}{K}\right]-\frac{a x(t) y(t)}{1+\omega x(t)}, \\
& \frac{d y(t)}{d t}=\frac{c x(t) y(t)}{1+\omega x(t)}-d y(t),
\end{aligned}
$$

$$
\begin{aligned}
& x\left(t^{+}\right)=\left(1-\frac{\delta x(t)}{x(t)+h}\right) x(t), \\
& y\left(t^{+}\right)=y(t)+\frac{\tau}{1+\theta y(t)},
\end{aligned}
$$$$
t=n T,
$$

where $\delta>0$ and $h>0$ represent the maximal fatality rate and the half-saturation constant for the prey, $\tau>0$ is the maximal release amount of the predator, and $\theta$ is a shape parameter. We assume that the control measures are conducted every $T$ time, and thus when $t=n T$ the amounts of the prey and the predator are updated to $(1-\delta x(t) /(x(t)+h)) x(t)$ and $y(t)+$ $\tau /(1+\theta y(t))$ instantaneously. Note that the function $\tau /(1+$ $\theta y(t))$ representing the amount of predators releasing at $t=$ $n T$ is a decreasing function with respect to $y(t)$. Note that the amount of natural enemies releasing at $t=n T$ depends on its density $y(n T)$, which indicates that the larger the density of natural enemy is, the smaller the natural enemy has been released at time $t=n T$, and obviously this strategy is more effective than the constant releasing method $[3,4,16,24]$.

The main purpose of the present paper is to focus on how the density dependent killing rate and density dependent releasing strategy affect the dynamics of the system and the effectiveness of the control measures. Based on this purpose, we first investigate the threshold condition which guarantees the existence and global stability of pest-free periodical solution. The sensitivity analyses for the key parameters on the threshold condition depict that reducing the pulse period may not be of benefit to the eradication of pests when the density dependent releasing method has been implemented, and this interesting result revealed by the proposed model shows that incorrect control methods may result in pest outbreak and resurgence. Furthermore, bifurcation analyses indicate that the forward and backward bifurcations could occur once the pest-free periodic solution becomes unstable, and the system could exist with two stable positive periodic solutions and even more complex dynamics. The important results found in this work confirm that the control actions should be carefully designed once the nonlinear impulsive control measures have been taken for pest management.

\section{Mathematical Analysis and Threshold Conditions}

In this section, we first demonstrate the existence of the pestfree periodic solution of model (3) and focus on its stability.

2.1. Existence and Stability of the Pest-Free Periodic Solution. In order to investigate the existence of the pest-free periodic solution, we first focus on the pest-free set $\left\{(x, y) \in \mathfrak{R}_{+}^{2}, x=\right.$ $0\}$, which is invariantly related to model (3). Within this set, model (3) becomes

$$
\frac{d y(t)}{d t}=-d y(t), \quad t \neq n T
$$




$$
\begin{aligned}
& y\left(t^{+}\right)=y(t)+\frac{\tau}{1+\theta y(t)}, \quad t=n T, \\
& y\left(0^{+}\right)=y_{0} .
\end{aligned}
$$

Solving the system in interval $(n T,(n+1) T]$, one yields

$$
y\left((n+1) T^{+}\right)=y\left(n T^{+}\right) e^{-d T}+\frac{\tau}{1+\theta y\left(n T^{+}\right) e^{-d T}} .
$$

Denote $y\left(n T^{+}\right)=y_{n}$; then the equation can be rewritten as follows:

$$
y_{n+1}=y_{n} e^{-d T}+\frac{\tau}{1+\theta y_{n} e^{-d T}} \doteq f\left(y_{n}\right)
$$

This equation is the so-called stroboscopic map of model (4). It describes the relations of the number of natural enemies between any two successive pulse points. So, the existence of the positive steady state of this equation implies the existence of a positive periodic solution of model (4). Thus, the steady state of $(6)$ is discussed first. The derivative of $f\left(y_{n}\right)$ with respect to $y_{n}$ can be given as follows:

$$
f^{\prime}\left(y_{n}\right)=e^{-d T}\left(1-\frac{\tau \theta}{\left(1+\theta y_{n} e^{-d T}\right)^{2}}\right) .
$$

Denote $y^{*}$ to be the fixed point of the stroboscopic map (6); then $y^{*}$ satisfies the equation $f\left(y^{*}\right)=y^{*} e^{-d T}+\tau /(1+$ $\left.\theta y^{*} e^{-d T}\right)=y^{*}$. So, $y^{*}$ satisfies the following equation:

$$
A_{1}\left(y^{*}\right)^{2}+A_{2} y^{*}+A_{3}=0
$$

where $A_{1}=\theta e^{-d T}\left(1-e^{-d T}\right), A_{2}=1-e^{-d T}$, and $A_{3}=-\tau$. Denote $\Delta=A_{2}^{2}-4 A_{1} A_{3}$; then we have

$$
\Delta=\left(1-e^{-d T}\right)^{2}+4 \tau \theta e^{-d T}\left(1-e^{-d T}\right)>0 .
$$

So, (6) has a unique positive root:

$$
y^{*}=\frac{-\left(1-e^{-d T}\right)+\sqrt{\Delta}}{2 \theta e^{-d T}}
$$

which is stable provided

$$
\left|f^{\prime}\left(y_{n}\right)\right|_{y_{n}=y^{*}}|=| e^{-d T}\left(1-\frac{\tau \theta}{\left(1+\theta y^{*} e^{-d T}\right)^{2}}\right) \mid<1 .
$$

Obviously, $e^{-d T}\left(1-\tau \theta /\left(1+\theta y^{*} e^{-d T}\right)^{2}\right)<1$. Moreover, $\left(1+\theta y^{*} e^{-d T}\right)^{2}=\tau \theta e^{-d T}+(1 / 2)\left(e^{-d T}-1\right)^{2}+(1 / 2)\left(e^{-d T}-\right.$ 1) $\sqrt{\Delta}+e^{-d T}+\sqrt{\Delta}$, which indicates that

$$
\begin{aligned}
& \frac{\tau \theta}{\left(1+\theta y^{*} e^{-d T}\right)^{2}} \\
& =\frac{\tau \theta}{\tau \theta e^{-d T}+(1 / 2)\left(e^{-d T}-1\right)^{2}+(1 / 2)\left(e^{-d T}-1\right) \sqrt{\Delta}+e^{-d T}+\sqrt{\Delta}} \\
& <e^{d T} .
\end{aligned}
$$

Therefore,

$$
e^{-d T}\left(1-\frac{\tau \theta}{\left(1+\theta y^{*} e^{-d T}\right)^{2}}\right)>e^{-d T}-1>-1
$$

and consequently $y^{*}$ is stable. Then, we can conclude that model (4) has a unique nontrivial positive periodic solution, denoted by $y_{p}(t)$, which can be expressed as follows:

$$
y_{p}(t)=y^{*} e^{-d(t-n T)}, \quad \text { for } n T<t \leq(n+1) T \text {. }
$$

Further, $y_{p}(t)$ is locally stable because $y^{*}$ is stable [25]. Then, we can obtain the following theorem by proving the global attractivity of $y^{*}$.

Theorem 1. Model (4) has a positive periodic solution $y_{p}(t)$ with period $T$ and for every solution $y(t)$ of $(4)$ one has $\mid y(t)-$ $y_{p}(t) \mid \rightarrow 0$ as $t \rightarrow+\infty$, where $y_{p}(t)=y^{*} e^{-d(t-n T)}(n T<t \leq$ $(n+1) T)$ and $y^{*}=\left(-\left(1-e^{-d T}\right)+\sqrt{\Delta}\right) / 2 \theta e^{-d T}$.

Proof. Let $f(y)=y e^{-d T}+\tau /\left(1+\theta y e^{-d T}\right)$. To study the properties of the function $f(y)$, we first solve the equation $d f(y) / d y=e^{-d T}\left(1-\tau \theta /\left(1+\theta y e^{-d T}\right)^{2}\right)=0$, with respect to $y$, and get two stationary points

$$
\begin{aligned}
& y_{1}=\frac{-\sqrt{\tau \theta}-1}{\theta} e^{d T}<0, \\
& y_{2}=\frac{\sqrt{\tau \theta}-1}{\theta} e^{d T} .
\end{aligned}
$$

The second derivative of the function $f(y)$ is $d^{2} f / d y^{2}=$ $2 \tau\left(\theta e^{-d T}\right)^{2} /\left(1+\theta y e^{-d T}\right)^{3}$. It is obvious that $\left(d^{2} f / d y^{2}\right)\left(y_{1}\right)<0$ and $\left(d^{2} f / d y^{2}\right)\left(y_{2}\right)>0$. So, $y_{1}$ is the local maximum and $y_{2}$ is the local minimum of the function $f(y)$ with $y_{2}>y_{1}$ and $y_{1}<0$.

Thus, if $y_{2}>0$, the function $f$ must be a convex function and if $y_{2}<0 f$ must be monotonous in the first quadrant. So, based on the sign of $y_{2}$ and the positional relations between point $y_{2}$ and $y^{*}$, we consider the following three possible cases.

Case $A\left(y_{2}<0<y^{*}\right)$. For this case the function $f(y)$ is a monotonically increasing function for $y>y_{2}$. For any $y \in\left[0, y^{*}\right)$, according to $y<f(y)<y^{*}$ we know that $f^{k}(y)$ is monotonically increasing as $k$ increases, and $f^{k}(y) \rightarrow$ $y^{*}(k \rightarrow \infty)$.

For any $y>y^{*}$, we have $f^{k}(y)>y^{*}$ for all $k$, and then according to $f(y)<y$ we conclude that $f^{k}(y)$ is monotonically decreasing as $k$ increases, which means that $f^{k}(y) \rightarrow y^{*}(k \rightarrow \infty)$.

Case $B\left(0<y_{2}<y^{*}\right)$. For this case, if $y \in\left[y_{2}, y^{*}\right)$ or $y>y^{*}$, then the conclusions corresponding to the Case A are true; that is, we have $f^{k}(y) \rightarrow y^{*}(k \rightarrow \infty)$. 
Moreover, for any $y \in\left[0, y_{2}\right)$, there must be $f(y)>$ $y_{2}$, and according to the conclusion of Case A we obtain $f^{1+k}(y) \rightarrow y^{*}(k \rightarrow \infty)$.

Case $C\left(y_{2}>y^{*}>0\right)$. For this case, it is easy to know that the function $f(y)$ is a monotonically decreasing function for $y \in\left[0, y_{2}\right]$, and it is an increasing function for $y \in\left(y_{2}, \infty\right)$.

It is easy to see that there must exist a positive integer $l$ such that $f^{l}(y) \in\left[y^{*}, y_{2}\right]$ for $y \in\left[0, y^{*}\right)$ or $y \in\left(y_{2}, \infty\right)$. Thus if $f^{k}(y) \rightarrow y^{*}(k \rightarrow \infty)$ for any $y \in\left(y^{*}, y_{2}\right]$, then $f^{n}(y) \rightarrow$ $y^{*}(n \rightarrow \infty)$ for any $y \in[0, \infty)$ and the results follow. Hence in the following we will focus on $f^{k}(y) \rightarrow y^{*}(k \rightarrow \infty)$ for any $y \in\left(y^{*}, y_{2}\right]$.

According to the definition of the function $f$ and by simple calculations, we have

$$
\begin{gathered}
f(y)=y e^{-d T}+\frac{\tau}{1+\theta y e^{-d T}} \\
f(f(y))=f(y) e^{-d T}+\frac{\tau}{1+\theta f(y) e^{-d T}}, \\
f^{\prime}(y)=e^{-d T}\left(1-\frac{\tau \theta}{\left(1+\theta y e^{-d T}\right)^{2}}\right), \\
f^{\prime}(f(y))=e^{-d T}\left(1-\frac{\tau \theta}{\left(1+\theta f(y) e^{-d T}\right)^{2}}\right) .
\end{gathered}
$$

Now we first show that $f^{\prime}(f(y))>-f(f(y)) / f(y)$ for any $y \in\left(y^{*}, y_{2}\right]$. In fact, for $y \in\left(y^{*}, y_{2}\right], f^{\prime}(f(y))>$ $-f(f(y)) / f(y) \Leftrightarrow f^{\prime}(f(y)) f(y)>-f(f(y))$, since

$$
\begin{aligned}
& f^{\prime}(f(y)) f(y) \\
& \quad=\left[e^{-d T}\left(1-\frac{\tau \theta}{\left(1+\theta f(y) e^{-d T}\right)^{2}}\right)\right] f(y), \\
& -f(f(y))=-f(y) e^{-d T}-\frac{\tau}{1+\theta f(y) e^{-d T}}, \\
& 2 f(y) e^{-d T}>0>\frac{-\tau}{\left(1+\theta f(y) e^{-d T}\right)^{2}},
\end{aligned}
$$

which indicates that

$$
f^{\prime}(f(y)) f(y)>-f(f(y)) .
$$

Next, we show that $f^{\prime}(y)>-f(y) / y$ for any $y \in\left(y^{*}, y_{2}\right]$. In fact, for $y \in\left(y^{*}, y_{2}\right]$ we have $f^{\prime}(y)>-f(y) / y \Leftrightarrow$ $f^{\prime}(y) y>-f(y)$. Since

$$
\begin{aligned}
f^{\prime}(y) y & =\left[e^{-d T}\left(1-\frac{\tau \theta}{\left(1+\theta y e^{-d T}\right)^{2}}\right)\right] y, \\
-f(y) & =-y e^{-d T}-\frac{\tau}{1+\theta y e^{-d T}}, \\
2 q_{2} e^{-d T} y & >0>\frac{-\tau}{\left[1+\theta y e^{-d T}\right]^{2}}
\end{aligned}
$$

thus we have

$$
f^{\prime}(f(y)) y>-f(y)
$$

It follows from $f^{\prime}(f(y))>-f(f(y)) / f(y)$ that we know

$$
f^{\prime}(f(y)) f^{\prime}(y) y<-\frac{f^{\prime}(y) y}{f(y)} f(f(y))
$$

and $f^{\prime}(y) y>-f(y)$ for $f^{\prime}(y)>-f(y) / y$; that is, $-f^{\prime}(y) y / f(y)<1$ holds. Furthermore, we have $0<$ $-f^{\prime}(y) y / f(y)<1$ due to $-f^{\prime}(y) y / f(y)>0$. All those confirm that $f^{\prime}(f(y)) f^{\prime}(y) y<-\left(f^{\prime}(y) y / f(y)\right) f(f(y))<$ $f(f(y))$; namely,

$$
f^{\prime}(f(y)) f^{\prime}(y) y<f(f(y)) .
$$

Denote $g(y)=f(f(y)) / y$; then we have $g^{\prime}(y)=$ $f^{\prime}(f(y)) f^{\prime}(y) y-f(f(y)) / y^{2}$. Then, $g^{\prime}(y)<0$ and $g(y)$ is a monotonically decreasing function at interval $y \in\left(y^{*}, y_{2}\right]$ due to (22).

Therefore, $g\left(y^{*}\right)=f\left(f\left(y^{*}\right)\right) / y^{*}=f\left(y^{*}\right) / y^{*}=y^{*} / y^{*}=$ 1 , and $g(y)<1$ for $y \in\left(y^{*}, y_{2}\right]$; that is, $f(f(y))<y$.

Based on the above discussions we conclude that $f^{2 k}(y)$ is a monotonically decreasing function as $k$ increases with $f^{2 k}(y) \rightarrow y^{*}(k \rightarrow \infty)$. Similarly, we can prove that $f^{2 k+1}(y)$ is a monotonically decreasing function as $k$ increases with $f^{2 k}(y) \rightarrow y^{*}(k \rightarrow \infty)$. Thus, $f^{n}(y) \rightarrow y^{*}(n \rightarrow \infty)$ for any $y \in[0, \infty)$ holds.

Combined with all cases together we know that the unique equilibrium $y^{*}$ is globally stable if it exists, and consequently the periodic solution $y_{p}(t)$ of model (4) is globally stable. This completes the proof.

The stability of the positive periodic solution is determined by the stability of the positive equilibrium of the difference equation made by sequence of impulsive points; thus the positive periodic solution $y_{p}(t)$ of model (4) is globally stable according to Theorem 1 . Therefore, a general expression of unique pest-free periodic solution of model (3) over the interval $n T<t \leq(n+1) T$ for all $n \in N$ can be denoted by

$$
\left(0, y_{p}(t)\right)=\left(0, y^{*} e^{-d(t-n T)}\right)
$$

In the following, we focus on the stability of the pest-free periodic solution for model (3). Firstly, define

$$
(\bar{x}(t), \bar{y}(t))=(x(t), y(t))-\left(0, y_{p}(t)\right)
$$

and then $(\bar{x}(t), \bar{y}(t))$ represents the deviations form of the pest-free solution. According to model (3), the derivations of $(\bar{x}(t), \bar{y}(t))$ are

$$
\begin{aligned}
& \dot{\bar{x}}=r \bar{x}-\frac{r}{K} \bar{x}^{2}-\frac{a \bar{x}}{1+\omega \bar{x}}\left(\bar{y}+y_{p}(t)\right), \\
& \dot{\bar{y}}=\frac{c \bar{x}}{1+\omega \bar{x}}\left(\bar{y}+y_{p}(t)\right)-d \bar{y} .
\end{aligned}
$$


Assuming that $(\bar{x}(t), \bar{y}(t))$ are small enough, then the linear approximation of the deviation system around the periodic solution $\left(0, y_{p}(t)\right)$ can be obtained as follows:

$$
\begin{aligned}
& \dot{\bar{x}}=\left(r-a y_{p}(t)\right) \bar{x} \\
& \dot{\bar{y}}=c y_{p}(t) \bar{x}-d \bar{y} .
\end{aligned}
$$

Then, we have the following main result.

Theorem 2. The pest-free periodic solution $\left(0, y_{p}(t)\right)$ of model (3) is locally stable in the first quadrant provided that

$$
R_{1} \doteq \frac{r d T}{a y^{*}\left(1-e^{-d T}\right)}<1 .
$$

Proof. Let $\Phi(t)$ be the fundamental matrix of (26); then

$$
\Phi(T)=\left(\begin{array}{cc}
e^{\int_{0}^{T}\left(r-a y_{p}(t)\right) d t} & 0 \\
* & e^{-d T}
\end{array}\right)
$$

and $\Phi(0)=I$ is the identity matrix. The term $*$ is not required in the following analysis.

The resetting impulsive conditions of (3) become

$$
\begin{aligned}
\left(\begin{array}{l}
\bar{x}\left(n T^{+}\right) \\
\bar{y}\left(n T^{+}\right)
\end{array}\right) & =\left(\begin{array}{cc}
1 & 0 \\
0 & 1-\frac{\tau \theta}{\left(1+\theta y_{p}(n T)\right)^{2}}
\end{array}\right)\left(\begin{array}{l}
\bar{x}(n T) \\
\bar{y}(n T)
\end{array}\right) \\
& =B(n T)\left(\begin{array}{l}
\bar{x}(n T) \\
\bar{y}(n T)
\end{array}\right) .
\end{aligned}
$$

Thus, the Floquet multipliers of the matrix

$$
M=B(T) \Phi(T)
$$

are

$$
\begin{aligned}
& \lambda_{1}=e^{\int_{0}^{T}\left(r-a y_{p}(t)\right) d t} \\
& \lambda_{2}=\left(1-\frac{\tau \theta}{\left(1+\theta y_{p}(T)\right)^{2}}\right) e^{-d T} .
\end{aligned}
$$

It follows from the literature [26] that the pest-free solution is locally stable if and only if $\left|\lambda_{1}\right|<1$ and $\left|\lambda_{2}\right|<1$. The former inequality is equal to $R_{1} \doteq \operatorname{rdT}_{\operatorname{ayy}}{ }^{*}\left(1-e^{-d T}\right)<1$ and the latter inequality is true if and only if $R_{2} \doteq \mid(1-$ $\left.\tau \theta /\left(1+\theta y^{*} e^{-d T}\right)^{2}\right) e^{-d T} \mid<1$, which is true naturally based on the analysis in the previous section. Therefore, the pest-free periodic solution is locally stable when $R_{1}<1$.

2.2. Boundness and Permanence of System (3). According to the proof of Theorem 2, the pest-free periodic solution is unstable when $R_{1}>1$. So, next we aim to prove the permanence of the system if $R_{1}>1$. First we prove the boundness of system (3).
Theorem 3. There exists a constant $M_{0}>0$ such that $x(t) \leq$ $M_{0}$ and $y(t) \leq M_{0}$ for each solution of system (3) with all $t$ large enough.

Proof. Suppose $x(t)$ and $y(t)$ are any solution of system (3). Let $V(t)=(c / a) x(t)+y(t)$. Then, when $t \neq n T$,

$$
\frac{d V(t)}{d t}+\lambda V(t)=\frac{c}{a}(r+\lambda) x-\frac{c r}{a K} x^{2}-(\lambda-d) y
$$

When $0<\lambda \leq d$, the right hand of the equation is bounded; namely,

$$
\frac{d V(t)}{d t}+\lambda V(t) \leq M_{1}
$$

with $M_{1}>0$. When $t=n T$,

$$
V\left(t^{+}\right)=V(t)+\frac{\tau}{1+\theta y(t)}-\frac{c}{a} \frac{\delta x(t)}{h+x(t)} \leq V(t)+\tau .
$$

Considering the system

$$
\begin{aligned}
& \frac{d V(t)}{d t} \leq-\lambda V(t)+M_{1}, \quad t \neq n T, \\
& V\left(t^{+}\right) \leq V(t)+\tau, \quad t=n T,
\end{aligned}
$$

we have

$$
\begin{aligned}
V(t) \leq & \left(V(0)^{+}-\frac{M_{1}}{\lambda}\right) e^{-\lambda t}+\frac{\tau\left(1-e^{-n \lambda T}\right)}{1-e^{-\lambda T}} e^{-\lambda(t-n T)} \\
& +\frac{M_{1}}{\lambda}
\end{aligned}
$$

So, $V(t)$ is uniformly ultimately bounded. Hence, there exists a constant $M_{0}>0$ such that $x(t) \leq M_{0}$ and $y(t) \leq M_{0}$ for each solution of system (3) with all $t$ large enough. This completes the proof.

Theorem 4. System (3) is permanent provided $R_{1}>1$.

Proof. It follows from Theorem 1 that there exists an $m_{1}>0$ such that $y \geq m_{1}$ for $t$ large enough. Thus, in order to show the permanence of model (3) we shall next find a constant $m_{2}>0$, such that $x \geq m_{2}$ for $t$ large enough.

Considering the second equation of system (3), we have

$$
\begin{aligned}
& \frac{d y(t)}{d t} \leq-d y(t), \quad t \neq n T, \\
& y\left(t^{+}\right)=y(t)+\frac{\tau}{1+\theta y(t)}, \quad t=n T .
\end{aligned}
$$

Therefore, according to the comparison theorem and the analyses in the previous subsection, we have

$$
y(t) \leq y_{p}(t)+\varepsilon_{1}
$$


for $t>t_{1}$ and $\varepsilon_{1}>0$. Then, by the first equation of system (3) we have

$$
\begin{aligned}
& \frac{d x(t)}{d t} \geq r x(t)\left(1-\frac{x(t)}{K}\right)-a\left(y_{p}(t)+\varepsilon_{1}\right) x(t), \\
& \quad t \neq n T, \\
& x\left(t^{+}\right)=\left(1-\frac{\delta x(t)}{x(t)+h}\right) x(t), \quad t=n T .
\end{aligned}
$$

Considering the auxiliary system

$$
\begin{aligned}
& \frac{d u(t)}{d t}=r u(t)\left(1-\frac{u(t)}{K}\right)-a\left(y_{p}(t)+\varepsilon_{1}\right) u(t), \\
& \quad t \neq n T, \\
& u\left(t^{+}\right)=\left(1-\frac{\delta u(t)}{u(t)+h}\right) u(t), \quad t=n T
\end{aligned}
$$

and using the same method as system (4), we have

$$
u_{n+1}^{+}=u_{n+1}\left(1-\frac{\delta u_{n+1}}{u_{n+1}+h}\right)
$$

with

$$
u_{n+1}=\frac{u_{n}^{+} e^{\int_{0}^{T}\left(r-a\left(y_{p}(t)+\varepsilon_{1}\right)\right) d t}}{1+u_{n}^{+} \int_{0}^{T}(r / K) e^{\int_{0}^{t}\left(r-a\left(y_{p}(\xi)+\varepsilon_{1}\right)\right) d \xi} d t}
$$

Let $M_{1}=e^{\int_{0}^{T}\left(r-a\left(y_{p}(t)+\varepsilon_{1}\right)\right) d t}$ and $M_{2}=$ $\int_{0}^{T}(r / K) e^{\int_{0}^{t}\left(r-a\left(y_{p}(\xi)+\varepsilon_{1}\right)\right) d \xi} d t$; then $u_{n+1}=u_{n}^{+} M_{1} /\left(1+M_{2} u_{n}^{+}\right)$. Therefore, we have the following different equation with respect to $u_{n}^{+}$:

$$
\begin{aligned}
u_{n+1}^{+} & =\frac{u_{n}^{+} M_{1}}{1+M_{2} u_{n}^{+}}\left(1-\frac{\delta\left(u_{n}^{+} M_{1} /\left(1+M_{2} u_{n}^{+}\right)\right)}{h+u_{n}^{+} M_{1} /\left(1+M_{2} u_{n}^{+}\right)}\right) \\
& \doteq F\left(u_{n}^{+}\right) .
\end{aligned}
$$

Let $u^{*}$ be the steady state of the above difference equation, and by simple calculation we have

$$
a_{1}\left(u^{*}\right)^{2}+a_{2} u^{*}+a_{3}=0
$$

where $a_{1}=M_{2}^{2}+M_{2} M_{1}, a_{2}=M_{2}+M_{1}+M_{2} h-M_{2} M_{1}-$ $M_{1}^{2}+\delta M_{1}^{2}$, and $a_{3}=h\left(1-M_{1}\right)$. Obviously, $a_{1}>0$. Moreover, $a_{3}<0$ when $R_{1}>1$ for $\varepsilon_{1}$ sufficiently small, which shows that the above difference equation exists with a unique positive equilibrium $u^{*}$. Therefore, system (40) has only one positive periodic solution when $R_{1}>1$, denoted by $\bar{u}(t)$.

To study the stability of this periodic solution, we first investigate the properties of the function $F(u)=\left(M_{1} u /(1+\right.$ $\left.\left.M_{2} u\right)\right)\left(1-\delta M_{1} u /\left(h\left(1+M_{2} u\right)+M_{1} u\right)\right)$. Taking the derivation of $F(u)$ with respect to $u$, one yields

$$
F^{\prime}(u)=\frac{M_{1}\left[\left(h M_{2}+M_{1}\right) u+h\right]^{2}-\left(2 \delta M_{1}^{2} h M_{2}+\delta M_{2}^{3}\right) u^{2}-2 \delta M_{1}^{2} h u}{\left(1+M_{2} u\right)^{2}\left[\left(h M_{2}+M_{1}\right) u+h\right]^{2}}
$$

Let $F^{\prime}(u)=0$; then we get the following equation:

$$
b_{1} u^{2}+b_{2} u+b_{3}=0
$$

where $b_{1}=M_{1} M_{2}^{2} h^{2}+2(1-\delta) h M_{1}^{2} M_{2}+(1-\delta) M_{1}^{3}, b_{2}=$ $2 h^{2} M_{1} M_{2}+2(1-\delta) h M_{1}^{2}$, and $b_{3}=h^{2} M_{1}$. It follows from $0<\delta<1$ that $b_{1}>0, b_{2}>0$, and $b_{3}>0$. Moreover, we can prove that $b_{2}^{2}-4 b_{1} b_{3}=4 M_{1}^{4} h^{2} \delta(1-\delta)>0$. Therefore, the equation $F^{\prime}(u)=0$ does not exist with any positive root. Thus, $F^{\prime}(u)>0$ for all $u>0$; that is, the function $F(u)$ is a monotonically increasing function for all $u>0$. Further, it is easy to show that $F^{\prime}(0)=M_{1}$ according to the expression of $F^{\prime}(u)$ with $M_{1}>1$ for $R_{1}>1$ and $\varepsilon_{1}$ sufficiently small. All those confirm that the intersection angle of the function $F(u)$ and the horizontal coordinate is larger than $\pi / 4$. Therefore, the function image is as shown in Figure 1. Then, according to $u<F(u)<u^{*}$ we have the fact that $F^{k}(u)$ is monotonically increasing as $k$ increases for any $u \in\left[0, u^{*}\right)$, and $F^{k}(u) \rightarrow$ $u^{*}(k \rightarrow \infty)$; according to $F(u)<u$ we have the fact that $F^{k}(u)$ is monotonically decreasing as $k$ increases for any $u>u^{*}$, and $F^{k}(u) \rightarrow u^{*}(k \rightarrow \infty)$. Therefore, the equilibrium $u^{*}$ is globally stable.

Thus, the positive periodic solution $\bar{u}(t)$ is globally stable. Then, according to the comparison theory, $x(t) \geq u(t) \geq$ $\bar{u}(t)-\varepsilon_{2}$ for $t \geq t_{2}$. Let $m_{2}=\min (\bar{u}(t))-\varepsilon_{2}, \varepsilon_{2}>0$; then we have $x \geq m_{2}$ for $t$ large enough. Thus, system (3) is permanent provided $R_{1}>1$. This completes the proof.

2.3. Bifurcation of Positive Periodic Solution. In this section, we investigate the existence and stability of positive periodic solution of system (3) by employing the bifurcation analysis related to the pest-free periodic solution $X_{0}=\left(0, y_{p}(t)\right)[26]$.

Theorem 5. As the parameter $T$ passes through the critical value $T_{0}$ satisfying $e^{\int_{0}^{T_{0}}\left[r-a y_{p}(\xi)\right] d \xi}=1$, a nontrivial positive periodic solution appears near $X_{0}$. The bifurcation is supercritical provided $B C<0$, while it is subcritical if $B C>0$, where

$$
B=-\left[\left(r-a y_{p}\left(T_{0}\right)\right)+a d T_{0} y^{*}\left(T_{0}\right) \frac{C_{2} e^{-d T_{0}}}{1-C_{2} e^{-d T_{0}}}\right]
$$




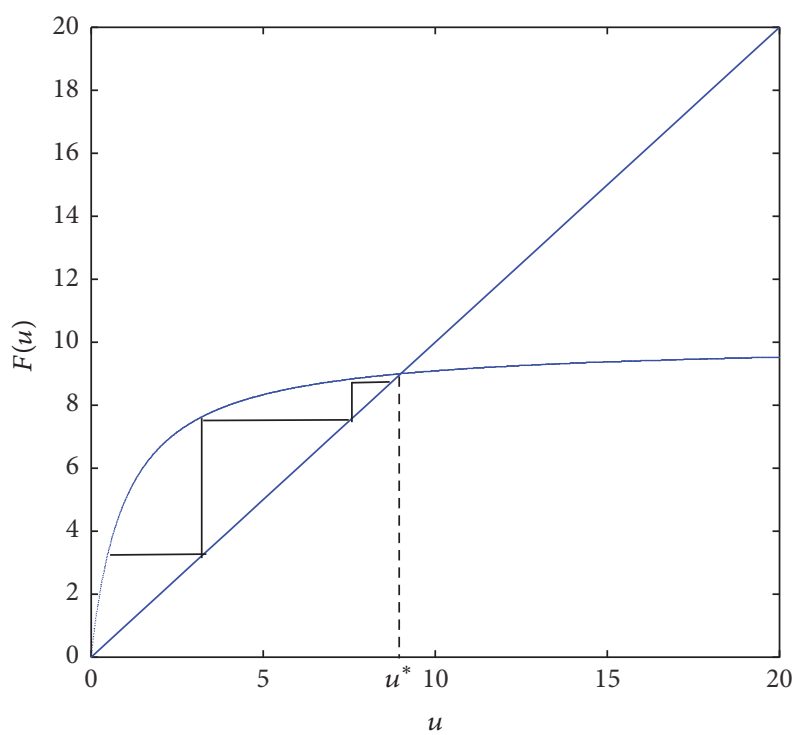

FIgURE 1: Illustration of the global stability of $u^{*}$.

$$
\begin{aligned}
& \cdot e^{\int_{0}^{T_{0}}\left[r-a y_{p}(\xi)\right] d \xi}, \\
C= & 2 \delta h^{-1} e^{2 \int_{0}^{T_{0}}\left[r-a y_{p}(\xi)\right] d \xi}-2 a T_{0} \frac{1-C_{1} C_{2}}{1-C_{2} e^{-d T_{0}}} \\
& \cdot e^{\int_{0}^{T_{0}}\left[r-a y_{p}(\xi)\right] d \xi}-C_{3} .
\end{aligned}
$$

Proof. To use the theory of [26], we employ the same notations as those in [26]; then

$$
\begin{aligned}
& F_{1}(x, y)=\frac{c x y}{1+\omega x}-d y, \\
& F_{2}(x, y)=r x\left[1-\frac{x}{K}\right]-\frac{a x y}{1+\omega x}, \\
& \Theta_{1}(x, y)=y+\frac{\tau}{1+\theta y}, \\
& \Theta_{2}(x, y)=\left(1-\frac{\delta x}{x+h}\right) x .
\end{aligned}
$$

By simple calculations, we get

$$
\begin{aligned}
\frac{\partial \Phi_{1}\left(T_{0}, X_{0}\right)}{\partial y} & =e^{-d T_{0}}>0, \\
\frac{\partial \Phi_{2}\left(T_{0}, X_{0}\right)}{\partial x} & =e^{\int_{0}^{T_{0}}\left[r-a y_{p}(\xi)\right] d \xi}>0, \\
\frac{\partial \Phi_{1}\left(T_{0}, X_{0}\right)}{\partial x} & =c \int_{0}^{T_{0}} y_{p}(u) e^{-d\left(T_{0}-u\right)} e^{\int_{0}^{u}\left[r-a y_{p}(\xi)\right] d \xi} d u \\
& \doteq C_{1}>0, \\
a_{0}^{\prime} & =1-C_{2} e^{-d T_{0}}, \\
d_{0}^{\prime} & =1-e^{\int_{0}^{T_{0}}\left[r-a y_{p}(\xi)\right] d \xi}, \\
b_{0}^{\prime} & =1-C_{1} C_{2},
\end{aligned}
$$

where $C_{2}=1-\tau \theta /\left(1+\theta y_{p}\left(T_{0}\right)\right)^{2}$ and $T_{0}$ is the root of $d_{0}^{\prime}=0$, satisfying the condition $c y^{*}\left(e^{-d\left(T_{0}-n T\right)}-1\right) / a d T_{0}=1$. Obviously, we have the fact that $C_{2}<1$, which shows that $a_{0}^{\prime}>0$. Further, we have

$$
\begin{aligned}
& \frac{\partial \Phi_{1}\left(T_{0}, X_{0}\right)}{\partial \bar{T}}=-d y^{*}\left(T_{0}\right) e^{-d T_{0}}, \\
& \frac{\partial^{2} \Phi_{2}\left(T_{0}, X_{0}\right)}{\partial x \partial y}=-a T_{0} e^{\int_{0}^{T_{0}}\left[r-a y_{p}(\xi)\right] d \xi}<0, \\
& \frac{\partial^{2} \Phi_{2}\left(T_{0}, X_{0}\right)}{\partial x \partial \bar{T}}=\left(r-a y_{p}\left(T_{0}\right)\right) e^{\int_{0}^{T_{0}}\left[r-a y_{p}(\xi)\right] d \xi}, \\
& \frac{\partial^{2} \Phi_{2}\left(T_{0}, X_{0}\right)}{\partial x^{2}}=T_{0}\left(-\frac{2 r}{K}+2 a \omega y_{p}\left(T_{0}\right)\right) \\
& \cdot e^{\int_{0}^{T_{0}}\left[r-a y_{p}(\xi)\right] d \xi} \\
& -\int_{0}^{T_{0}} a e^{\int_{u}^{T_{0}}\left[r-a y_{p}(\xi)\right] d \xi}\left[\int_{0}^{u} e^{-d(u-v)} c y_{p}(v)\right. \\
& \left.\cdot e^{\int_{0}^{T_{0}}\left[r-a y_{p}(\xi)\right] d \xi} d v\right] d u \doteq C_{3} .
\end{aligned}
$$

$\partial \Theta_{1}\left(T_{0}, X_{0}\right) / \partial y=C_{2}, \partial \Theta_{1}\left(T_{0}, X_{0}\right) / \partial x=0, \partial \Theta_{2} / \partial x=1$, $\partial \Theta_{2}\left(T_{0}, X_{0}\right) / \partial y=0, \partial^{2} \Theta_{1}\left(T_{0}, X_{0}\right) / \partial x \partial y=-2 \delta h^{-1}$, and $\partial^{2} \Theta_{1}\left(T_{0}, X_{0}\right) / \partial x \partial y=0$. Thus,

$$
\begin{aligned}
B= & -\left[\left(r-a y_{p}\left(T_{0}\right)\right)+a d T_{0} y^{*}\left(T_{0}\right) \frac{C_{2} e^{-d T_{0}}}{1-C_{2} e^{-d T_{0}}}\right] \\
& \cdot e^{\int_{0}^{T_{0}}\left[r-a y_{p}(\xi)\right] d \xi}, \\
C= & 2 \delta h^{-1} e^{2 \int_{0}^{T_{0}}\left[r-a y_{p}(\xi)\right] d \xi}-2 a T_{0} \frac{1-C_{1} C_{2}}{1-C_{2} e^{-d T_{0}}} \\
& \cdot e^{\int_{0}^{T_{0}}\left[r-a y_{p}(\xi)\right] d \xi}-C_{3} .
\end{aligned}
$$

Therefore, according to Theorem 2 in literature [26], the supercritical branch occurs at the point $T_{0}$ if $B C<0$ and the subcritical branch occurs if $B C>0$. This completes the proof.

\section{Numerical Investigations}

Bifurcation analyses shown above indicate that system (3) could exist with complex dynamics once the pest-free periodic solution becomes unstable, which is a challenge for theoretical studies. Therefore, in this section we will focus on the complex dynamics by using numerical techniques.

3.1. Backward and Forward Bifurcation. Our theoretical analysis shows that when $R_{1}>1$ system (3) is permanent and when $R_{1}<1$ the pest-free periodic solution is locally stable in the first quadrant. Moreover, Theorem 5 indicates the existence of nontrivial positive periodic solution under certain conditions. Thus, to verify these theoretical results, we 


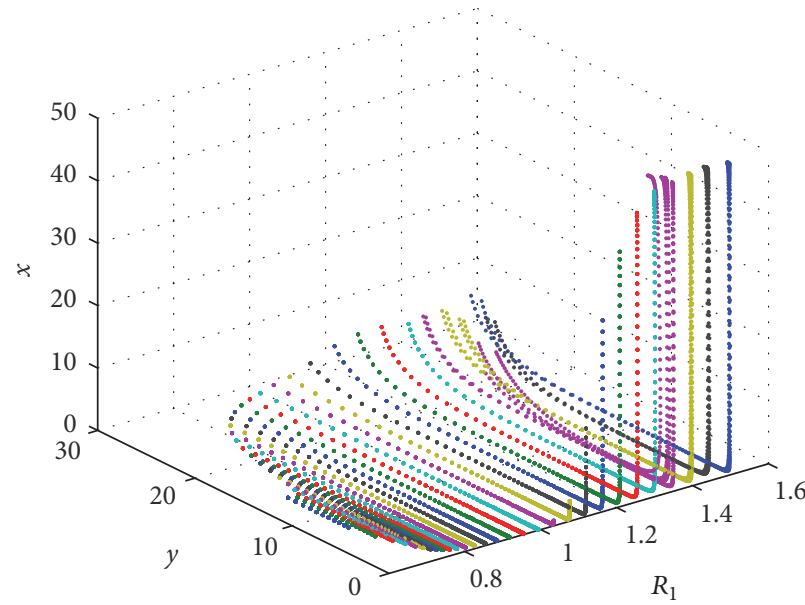

(a)

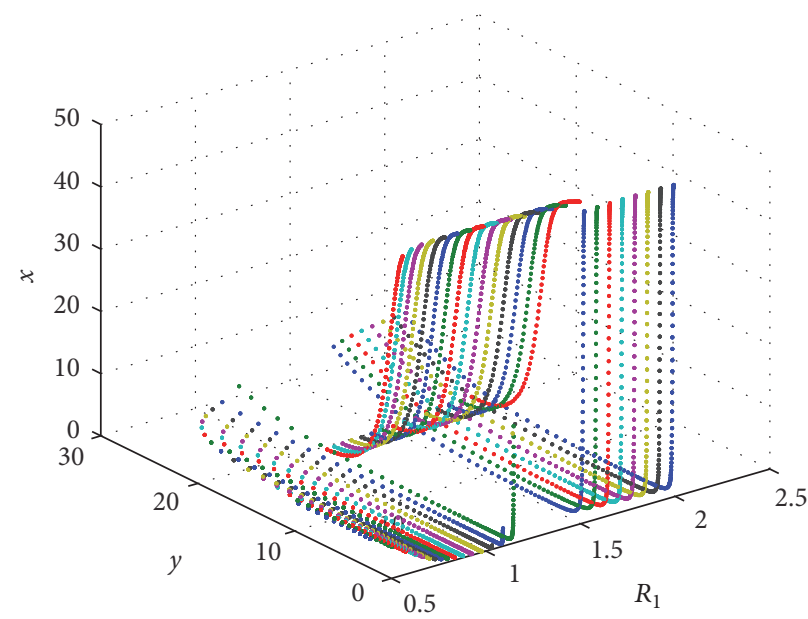

(b)

Figure 2: Backward (forward) bifurcation of system (3) with respect to threshold value $R_{1}$. The parameter values are as follows: $T=6, \tau=$ 30, $\theta=0.5, \delta=0.85, h=0.8, r=1.5, a=0.3, K=50, \omega=0.3, c=0.23$, and $d=0.6$ in (a) and $d=0.8$ in (b).

first take some numerical simulations under certain parameter spaces and initial values. Figure 2 shows the bifurcation diagram of system (3) with respect to the threshold condition $R_{1}$ with $d=0.6$ and $d=0.8$.

It follows from Figure 2(a) that when $R_{1}<1$ there exists a stable pest-free periodic solution, and when $R_{1}>1$ the pest-free periodic solution becomes unstable and a stable positive periodic solution appears, which is called a forward bifurcation at $R_{1}=1$. Figure 2(b) indicates that when $R_{1}<$ $0.78(T<0.406)$ there exists only one pest-free periodic solution and it is stable. When $R_{1}>1$, there exists at least one stable positive periodic solution. Specifically, when $0.78<$ $R_{1}<1$, the pest-free periodic and the positive periodic solutions can coexist which are bistable; namely, when the value of $R_{1}$ passes through 1 the supercritical bifurcation occurs or backward bifurcation occurs. Both of these two figures confirm that when $R_{1}<1$ the pest-free periodic solution is locally stable and when $R_{1}>1$ system (3) is permanent. Moreover, there may also exist a locally stable positive periodic solution when $R_{1}<1$, and this interesting result could be produced by the density dependent pulse control strategies.

As shown in Figure 2(b), there may be two locally stable periodic solutions when $R_{1}<1$. Therefore, the initial values of both the pest and predator populations are crucial. To see how different initial values affect the successful pest control, we plot the basins of attraction of two locally stable periodic solutions of system (3), as shown in Figure 2(b). It follows from Figure 3 that the pest can be eradicated when the initial values of the predator are very small or large. However, when the initial values of the predator have been chosen between 2 and 30 , the pest can be eradicated only if the initial values of the pest are very small.

The theoretical results only give the existence and global stability of the pest-free periodic solution or local stability of the positive periodic solution. To further study the dynamics of system (3), the bifurcation diagrams for the

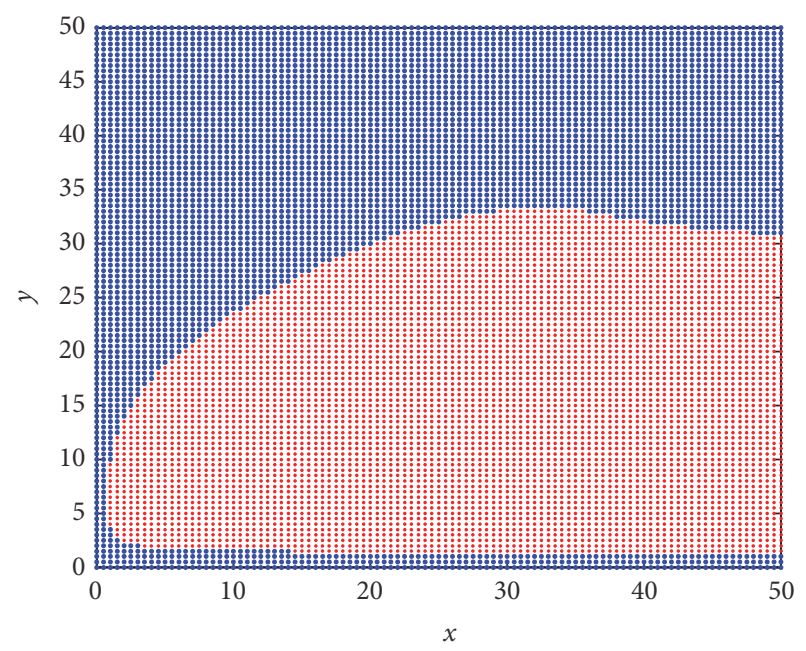

Figure 3: Basins of attraction of system (3). The parameter values are as follows: $T=6, r=1.5 ; a=0.3 ; K=50 ; \omega=0.3 ; c=$ $0.23 ; d=0.8 ; \tau=30 ; h=0.8 ; \delta=0.85 ; \theta=0.5$.

pest population with respect to bifurcation parameters $T$, $\tau$, and $\delta$ are displayed in Figures 4, 5, and 6. The resulting bifurcation diagrams clearly show rich dynamics of system (3). Specifically, Figure 5 shows the existence of periodhalving bifurcations, and Figure 4 depicts the existence of period-doubling bifurcations, while Figure 6 indicates that several types of attractor may coexist. All those complex dynamics may result in complexity of pest control.

\subsection{Effects of Density Dependent Releasing Strategy. Accord-} ing to the main results shown in Theorem 4, system (3) is permanent provided $R_{1}>1$. Thus, to control the pest population, we need to know how the parameters affect the value of the threshold $R_{1}$. Obviously, the threshold condition is determined by the key parameters including the 


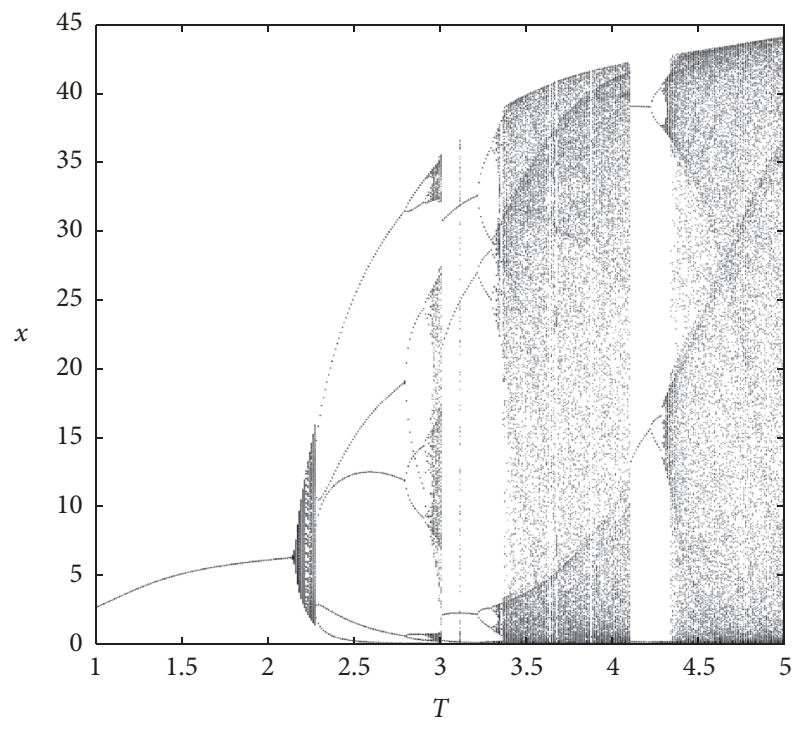

Figure 4: Bifurcation diagram of system (3) with respect to $T$. The parameter values are as follows: $\tau=1, \theta=0.5, \delta=0.85, h=$ $0.8, r=1.5, a=0.3, K=50, \omega=0.3, d=0.8, c=0.5$.

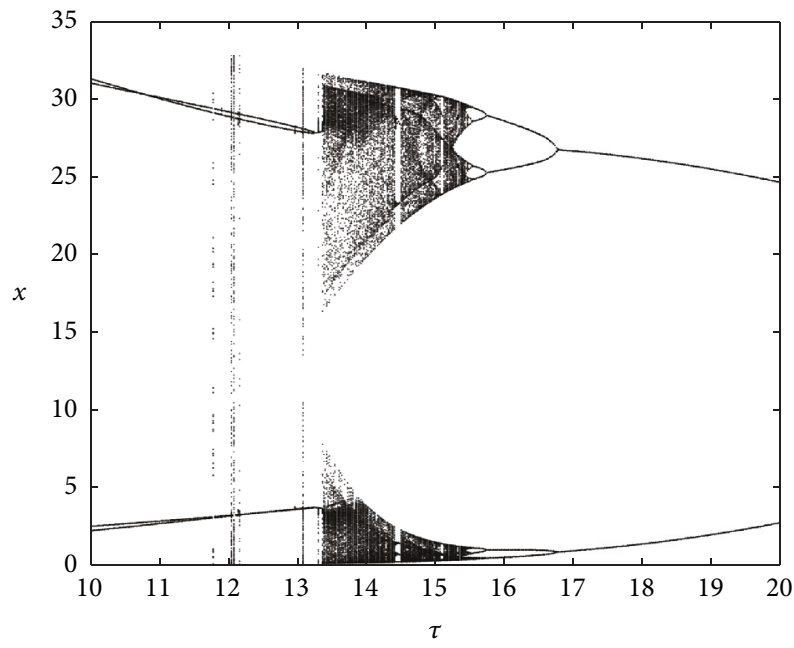

FIgURE 5: Bifurcation diagram of system (3) with respect to parameter $\tau$. The other parameter values are as follows: $T=6 \theta=$ $0.5, \delta=0.85, h=0.8, r=1.5, a=0.3, K=50, \omega=0.3, d=$ $0.8, c=0.5$.

pulse frequency, the maximum fatality rate, and the halfsaturation constant. Figure 7 shows the relationship between the threshold $R_{1}$ and the pulse period $T$. It follows from this figure that the value of $R_{1}$ could increase as pulse period $T$ increases, as shown in Figure 7(a). However, it also can decrease as $T$ increases, as shown in Figure 7(b). All those indicate that the relations between the threshold $R_{1}$ and pulse period $T$ are quite complex, which depicts the threshold condition $R_{1}$ is nonmonotonic with respect to $T$. Thus, reducing the pulse period $T$ is not always beneficial for pest control. That is, frequent releasing of natural enemies may not be beneficial to the eradication of pests when the amount of predator released depends on the predator population, which is one of the novel results obtained in the present work

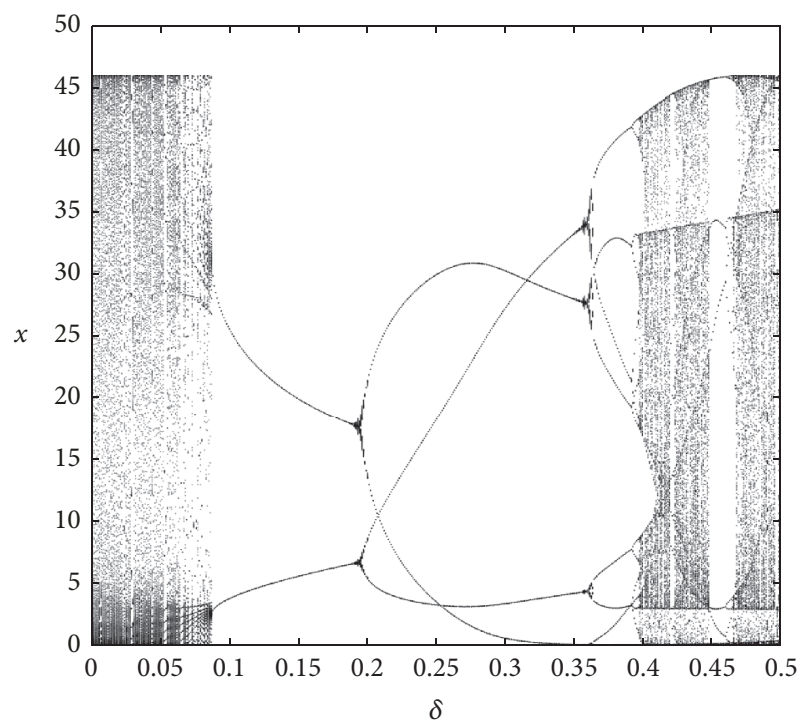

Figure 6: Bifurcation diagram of system (3) with respect to parameter $\delta$. The other parameter values are as follows: $T=6, \tau=$ $1, \theta=0.5, h=0.8, r=1.5, a=0.3, K=50, \omega=0.3, d=$ $0.8, c=0.5$.

compared with the previous results obtained on this topic [3$5,13,27]$.

The releasing term in model (3) is formulated by $\tau /(1+$ $\theta y)$, which is a predator density dependent function and depicts how the number of natural enemy populations released was guided by its current density at the fixed moment. The parameter $\theta$ is a shape parameter which indicates the dependence of releasing amount and the predator population. Thus, to investigate the effects of the predator population guided releasing method on the pest control, the variations of the threshold $R_{1}$ with respect to parameters $T$, $\theta$, and $\tau$ have been addressed, as shown in Figure 8 .

Figure $8(\mathrm{a})$ shows the contour plots of $R_{1}$ with respect to $\theta$ and $\tau$. It follows from this figure that increasing $\tau$ can lead to an obvious decrease of $R_{1}$, no matter how much the value of $\theta$ is, while decreasing $\theta$ can also lead to a slight decrease of $R_{1}$ and the effect is more evident when the value of $\tau$ is large. Figure 8(b) shows the contour plots of $R_{1}$ with respect to $T$ and $\tau$. This figure indicates that both $T$ and $\tau$ together can affect the threshold condition $R_{1}$ significantly. Note that for relatively small $\tau$ the threshold condition $R_{1}$ is a decreasing function as $T$ decreases. However, for a relatively large $\tau$, decreasing period $T$ may first lead to a complex effect on $R_{1}$; that is, $R_{1}$ is nonmonotonic with respect to $T$, which is similar to the important results shown in Figure 7.

Similarly, this type of interesting results can also be found in Figure 8(c) for the contour plots of $R_{1}$ with respect to $\theta$ and $T$. Figure $8(\mathrm{c})$ indicates that if $T$ is small, decreasing $\theta$ can result in an obvious decrease of $R_{1}$, while if $T$ is relatively large (larger than 8 ), decreasing $\theta$ almost has no effect on $R_{1}$. Thus, Figure 8 reveals that the key control factors including the pulse frequency, pulse amount, and the dependence of pulse amount and the current amount of the predator could affect each other. For example, when the control measure is implemented frequently (i.e., $T$ is small) or the releasing 


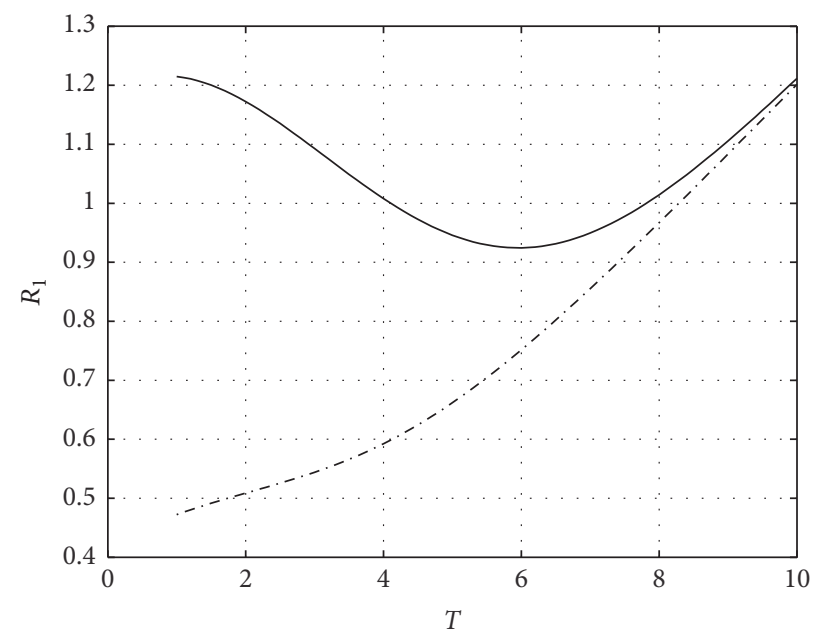

(a)

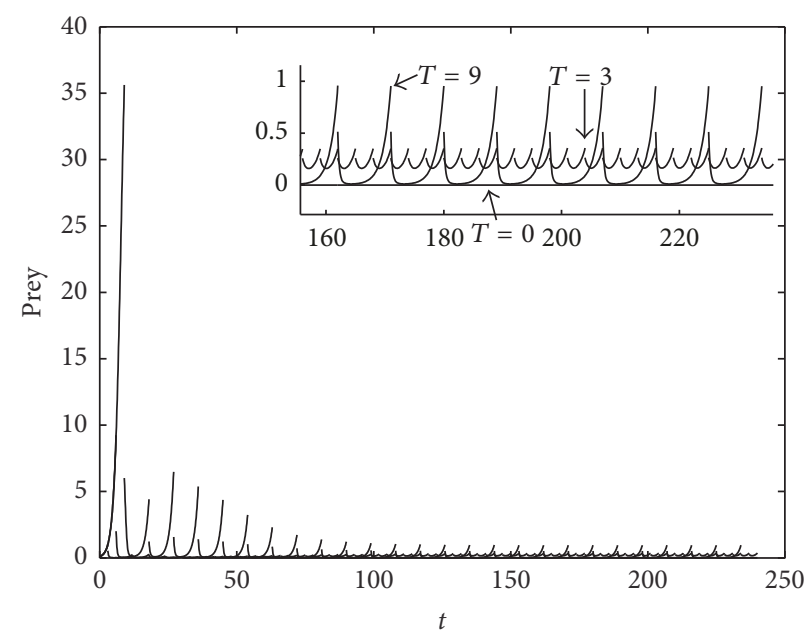

(b)

Figure 7: (a) Relationship between the threshold $R_{1}$ and the pulse period $T$ with $\theta=0.5$ (the dashed line) and $\theta=4$ (the solid line). (b) Behavior of the prey when $T=3,6$, and 9 for the case $\theta=4$. The values of $R_{1}$ when $T=3,6$, and 9 are $1.0928,0.9245$, and 1.1056 , respectively. Other parameter values are $r=0.8, a=0.3, \tau=20, d=0.9$.

amount is large (i.e., $\tau$ is large), effect of the dependence between the pulse amount and the current amount of the predator is very small. Moreover, the complex relationships between $T$ and $R_{1}$ usually appear when $\tau$ and $\theta$ are relatively large. All those confirm the control measures should be carefully designed in practice. Otherwise, the paradox and hormesis could occur when pesticides are applied in the field.

\section{Discussion}

Considering the effects of resources limitation and pest management purpose, we proposed a Holling type II pest-natural enemy model with density guided releasing strategy and killing rate, which can provide a more nature understanding and a better portrayal of the reality. However, the nonlinear pulse functions not only result in difficulties for dynamic analyses, but also can reveal some important results related to pest control compared with those obtained in the previous papers $[3,4,9,22,23]$.

Theoretical results show that the pest-free periodic solution is locally stable when $R_{1}<1$ and the system is permanent when $R_{1}>1$. Besides, theoretical analysis also indicates that when $T$ passes through the critical value $T_{0}$, a nontrivial positive periodic solution appears near the pest-free periodic solution. Further, extensive numerical investigations imply that there may be a locally stable positive periodic solution even if $R_{1}<1$. This results in a backward bifurcation and is quite different from the previous works $[3,17]$, in which the pulse functions are linear. This also indicates that the pest may not be eradicated even if $R_{1}$ is controlled to be below 1. All those results demonstrate that the dynamic behavior of model (3) may be dramatically affected by small changes in the value of initial densities of pest and natural enemy under resource limitation. Moreover, as shown in Figure 3, small or large initial values of the natural enemies may lead to the eradication of the pest. Medium level of the initial values of the natural enemies usually can not suppress the pest population and often lead to the outbreak of the pest.

The threshold condition $R_{1}$ depends on all key parameters related to the pest control, such as releasing period, maximum releasing constant, and shape parameters of the nonlinear functions. To analyze the effects of those parameters, we first investigate the relationship between $R_{1}$ and the pulse period. Our results show that $R_{1}$ may be a nonmonotonic function with respect to the pulse period $T$. This indicates that shortening the pulse period may lead to an increase of $R_{1}$ and consequently may cause the outbreak of the pest, as shown in Figure 7. This result is not the same as the traditional results obtained in the previous works $[3,4,9,22,23]$. This may be because that frequent release leads to large number of current predators. As the number of predators released is guided by its current density, the large number of the current predators results in a small amount of releasing every time. So, under this circumstance, frequent release of the predator may be beneficial or detrimental to the eradication of pests.

The releasing number and killing rate guided by both the pest and natural enemy populations have been introduced into the model; one of the main purposes is to analyze the effect of this kind of nonlinear control methods in terms of reducing the pest population. So, it is essential to suggest how to choose the shape parameter $\theta$ and the releasing rate $\tau$ and to determine how the releasing period affects the control output. Numerical results as shown in Figure 8 reveal that when releasing rate is relatively large, reducing the dependence of releasing amount and the predator population, namely, reducing the value of $\theta$, has little effect on the reduction of the pest population, while it has large effect on the reduction of the population of the pest when the releasing rate is small. Moreover, frequent releasing of the predator can only lead to decreasing of $R_{1}$ when the dependence of releasing amount and the predator population is small and frequent releasing of the predator may result in an increase 


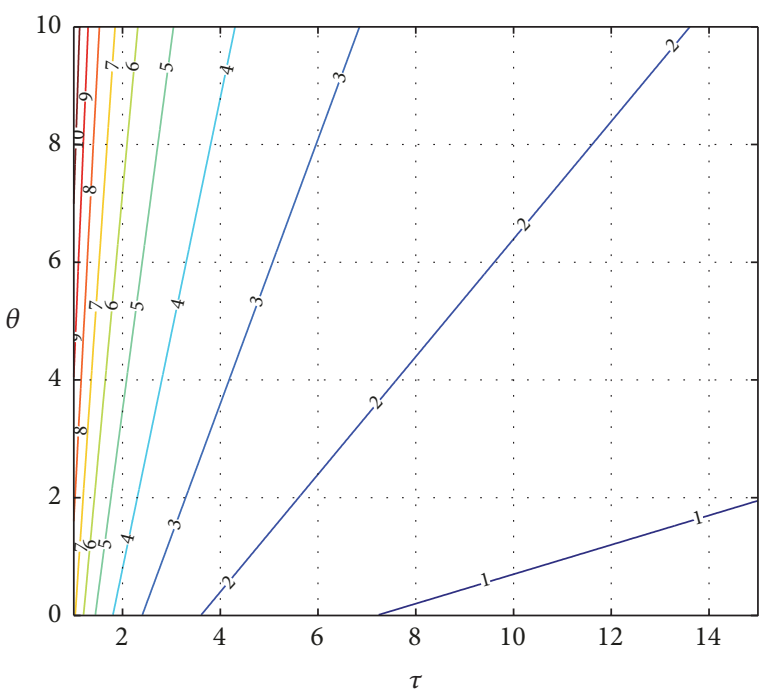

(a)

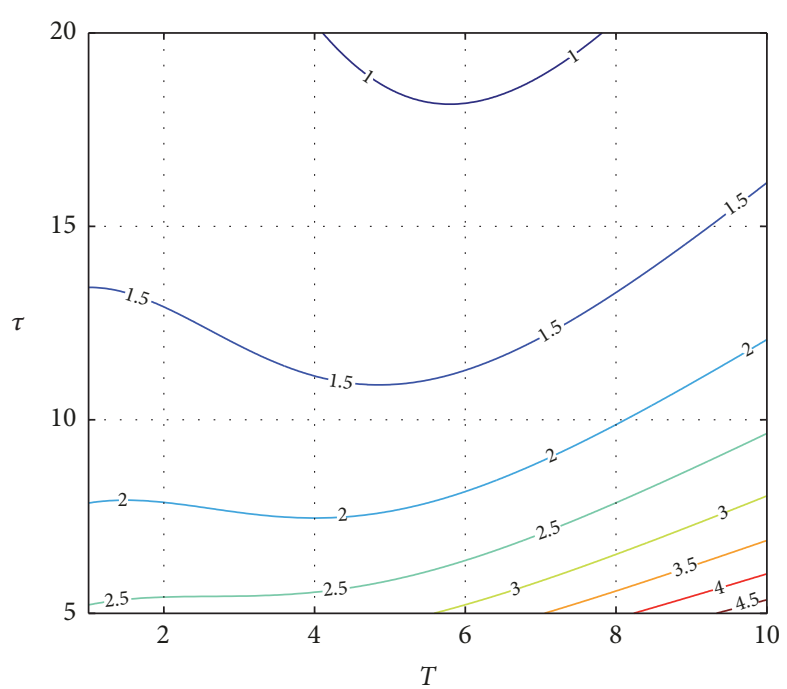

(b)

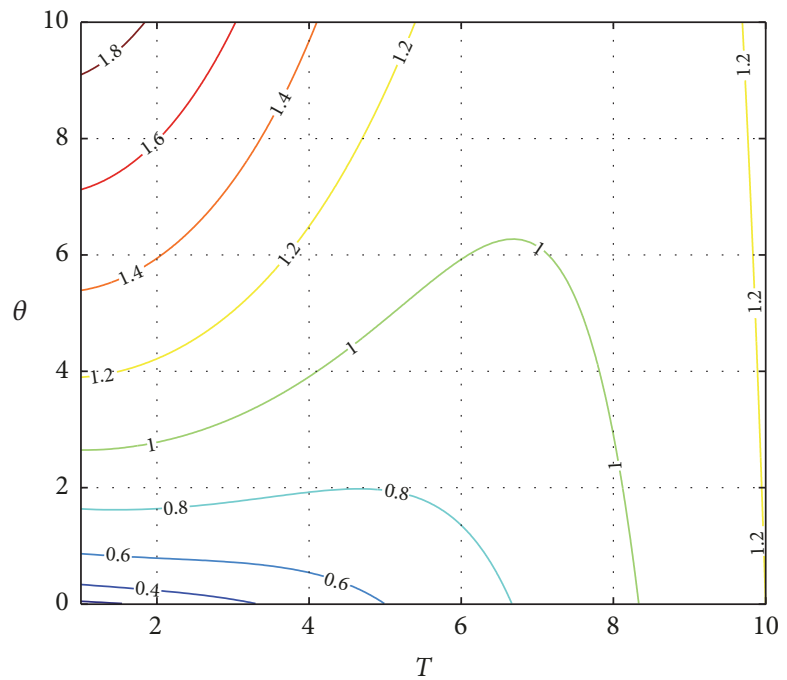

(c)

Figure 8: (a) Contour plots of $R_{1}$ with respect to $\theta$ and $\tau$ for $T=3$. (b) Contour plots of $R_{1}$ with respect to $\tau$ and $T$ when $\theta=4$. (c) Contour plots of $R_{1}$ with respect to $\theta$ and $T$ when $\tau=20$. The other parameters are fixed as $r=0.8, d=0.9$, and $a=0.3$.

of $R_{1}$ when the dependence of releasing amount and the predator population is large.

The results presented in this paper indicate that the successful biological control depends on the initial densities of the pest and predator and the reasonable implementing plan of control measures. The releasing of predators guided by the predator population is more sensitive to the pulse period and releasing amount every time. However, some simplifications of the real prey-predator interactions are neglected, such as predator hiding and effect of pesticide on the predator. In fact, the prey refuge can be broadly defined including spatial or temporal refuges, prey aggregations, or reduced prey activity [28-31]. It has been shown in many previous papers that the existence of refuges may have important effects on the coexistence of the prey and predator. Therefore, more realistic mathematical model and its dynamic analyses should be studied in the future which can help us to design control strategies and to make management decisions.

\section{Conflicts of Interest}

The authors declare that they have no conflicts of interest.

\section{Acknowledgments}

This work is supported by the National Natural Science Foundation of China (NSFC, 11471201, 11601301), by the Fundamental Research Fund for the Central Universities (GK201603003, GK201701001), by Young Talent Fund of University Association for Science and Technology in Shaanxi, China (20160212), and by General Financial Grant from the China Postdoctoral Science Foundation (2016M602758). 


\section{References}

[1] J. C. Van Lenteren, "Integrated pest management in protected crops," in Integrated Pest Management, pp. 311-320, Chapman \& Hall, London, UK, 1995.

[2] J. C. Van Lenteren and J. Woets, "Biological and integrated pest control in greenhouses," Annual Review of Entomology, vol. 33, no. 1, pp. 239-250, 1988.

[3] S. Tang, G. Tang, and R. A. Cheke, "Optimum timing for integrated pest management: modelling rates of pesticide application and natural enemy releases," Journal of Theoretical Biology, vol. 264, no. 2, pp. 623-638, 2010.

[4] S. Tang, W. Pang, R. A. Cheke, and J. Wu, "Global dynamics of a state-dependent feedback control system," Advances in Difference Equations, vol. 2015, no. 322, 2015.

[5] S. Tang, B. Tang, A. Wang, and Y. Xiao, "Holling II predatorprey impulsive semi-dynamic model with complex poincaré map," Nonlinear Dynamics, vol. 81, no. 3, pp. 1575-1596, 2015.

[6] H. K. Shaw, P. DeBach, and E. I. Schlinger, Biological Control of Insect Pests and Weeds, Reinhold Pub. Corp., New York, NY, USA, 1964.

[7] H. I. Freedman, "Graphical stability, enrichment, and pest control by a natural enemy," Mathematical Biosciences, vol. 31, no. 3-4, pp. 207-225, 1976.

[8] L. E. Caltagirone and R. L. Doutt, "The history of the vedalia beetle importation to California and its impact on the development of biological control," Annual Review of Entomology, vol. 34, pp. 1-16, 1989.

[9] S. Tang and R. A. Cheke, "State-dependent impulsive models of integrated pest management (IPM) strategies and their dynamic consequences," Journal of Mathematical Biology, vol. 50, no. 3, pp. 257-292, 2005.

[10] D. Bainov and P. Simeonov, Impulsive Differential Equations: Periodic Solutions and Applications, Longman Scientific and Technical, New York, NY, USA, 1993.

[11] V. Lakshmikantham, D. Bainov, and P. Simeonov, Theory of Impulsive Differential Equations, World Scientific, Singapore, 1989.

[12] S. Y. Tang, Y. N. Xiao, and R. A. Cheke, "Multiple attractors of host-parasitoid models with integrated pest management strategies: eradication, persistence and outbreak," Theoretical Population Biology, vol. 73, no. 2, pp. 181-197, 2008.

[13] S. Tang, J. Liang, Y. Tan, and R. A. Cheke, “Threshold conditions for integrated pest management models with pesticides that have residual effects," Journal of Mathematical Biology, vol. 66, no. 1-2, pp. 1-35, 2013.

[14] X. Liu and L. Chen, "Complex dynamics of holling type II lotkavolterra predator-prey system with impulsive perturbations on the predator," Chaos Solitons \& Fractals, vol. 16, no. 2, pp. 311$320,2003$.

[15] P. Georgescu, H. Zhang, and L. Chen, "Bifurcation of nontrivial periodic solutions for an impulsively controlled pest management model," Applied Mathematics and Computation, vol. 202, no. 2, pp. 675-687, 2008.

[16] J. Liang, S. Tang, and R. A. Cheke, "An integrated pest management model with delayed responses to pesticide applications and its threshold dynamics," Nonlinear Analysis. Real World Applications. An International Multidisciplinary Journal, vol. 13, no. 5, pp. 2352-2374, 2012.

[17] B. Liu, L. Chen, and Y. Zhang, "The dynamics of a preydependent consumption model concerning impulsive control strategy," Applied Mathematics and Computation, vol. 169, no. 1, pp. 305-320, 2005.

[18] B. Liu, Z. Teng, and L. Chen, "Analysis of a predator-prey model with holling II functional response concerning impulsive control strategy," Journal of Computational and Applied Mathematics, vol. 193, no. 1, pp. 347-362, 2006.

[19] G. Jiang and Q. Lu, "The dynamics of a prey-predator model with impulsive state feedback control," Discrete and Continuous Dynamical Systems. Series B. A Journal Bridging Mathematics and Sciences, vol. 6, no. 6, pp. 1301-1320, 2006.

[20] S. Shulin and G. Cuihua, "Dynamics of a beddington-deangelis type predator-prey model with impulsive effect," Journal of Mathematics, vol. 2013, Article ID 826857, 11 pages, 2013.

[21] Y. Du, R. Xu, and L. Duan, "Dynamics of a stage-structured predator-prey model concerning impulsive control strategy," Journal of Biological Systems, vol. 17, no. 4, pp. 779-792, 2009.

[22] W. Qin, S. Tang, and R. A. Cheke, "The effects of resource limitation on a predator-prey model with control measures as nonlinear pulses," Mathematical Problems in Engineering, vol. 2014, Article ID 450935, 13 pages, 2014.

[23] J. Yang and S. Tang, "Holling type II predator-prey model with nonlinear pulse as state-dependent feedback control," Journal of Computational and Applied Mathematics, vol. 291, pp. 225-241, 2016.

[24] S. Tang and J. Liang, "Global qualitative analysis of a nonsmooth Gause predator-prey model with a refuge," Nonlinear Analysis, vol. 76, pp. 165-180, 2013.

[25] P. Cull, "Local and global stability for population models," Biological Cybernetics, vol. 54, no. 3, pp. 141-149, 1986.

[26] A. Lakmeche and O. Arino, "Bifurcation of non trivial periodic solutions of impulsive differential equations arising chemotherapeutic treatment," Dynamics of Continuous, Discrete and Impulsive Systems, vol. 7, no. 2, pp. 265-287, 2000.

[27] S. Tang, J. Liang, Y. Xiao, and R. A. Cheke, "Sliding bifurcations of filippov two stage pest control models with economic thresholds," SIAM Journal on Applied Mathematics, vol. 72, no. 4, pp. 1061-1080, 2012.

[28] G. Tang, S. Tang, and R. A. Cheke, "Global analysis of a holling type II predator-prey model with a constant prey refuge," Nonlinear Dynamics, vol. 76, no. 1, pp. 635-647, 2014.

[29] E. González-Olivares and R. Ramos-Jiliberto, "Dynamic consequences of prey refuges in a simple model system: more prey, fewer predators and enhanced stability," Ecological Modelling, vol. 166, no. 1-2, pp. 135-146, 2003.

[30] W. Ko and K. Ryu, "Qualitative analysis of a predator-prey model with holling type II functional response incorporating a prey refuge," Journal of Differential Equations, vol. 231, no. 2, pp. 534-550, 2006.

[31] J. N. McNair, "The effects of refuges on predator-prey interactions: a reconsideration," Theoretical Population Biology. An International Journal, vol. 29, no. 1, pp. 38-63, 1986. 


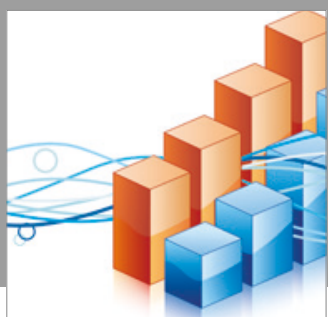

Advances in

Operations Research

vatersals

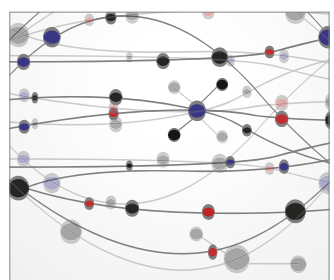

\section{The Scientific} World Journal
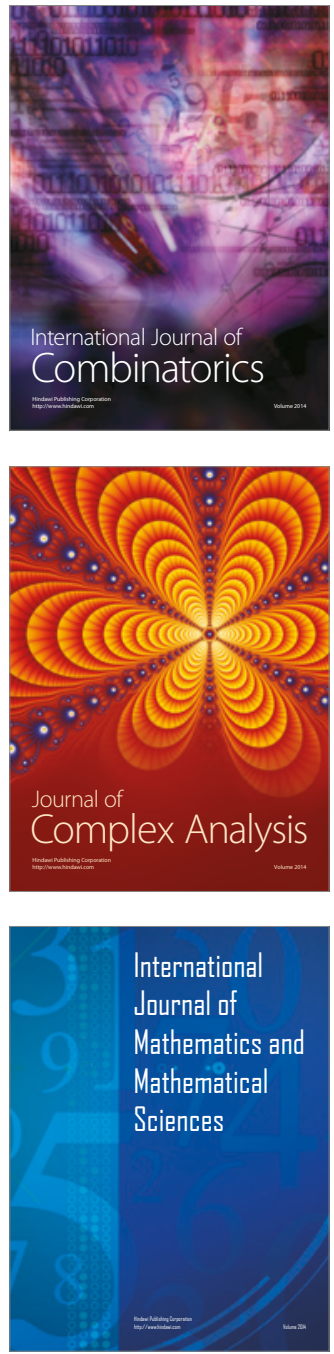
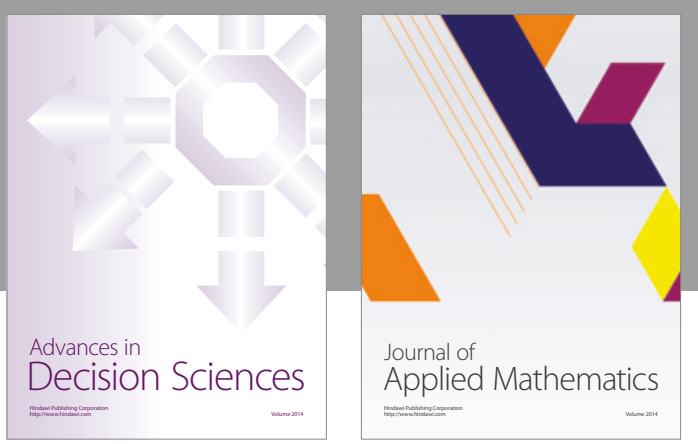

Algebra

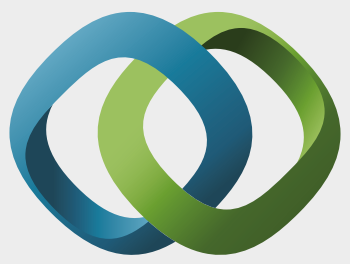

\section{Hindawi}

Submit your manuscripts at

https://www.hindawi.com
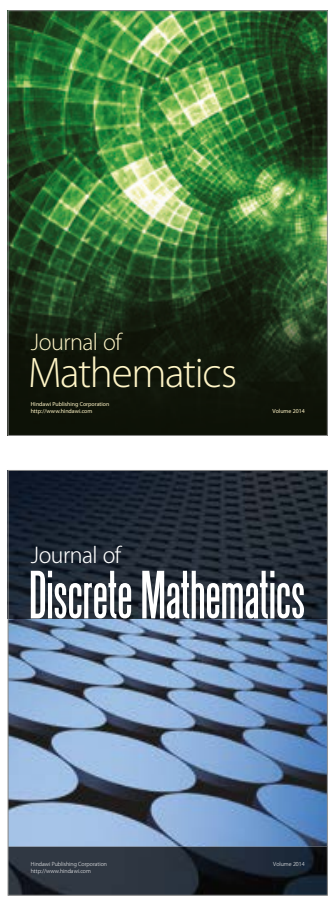

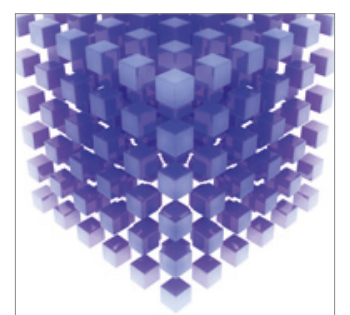

Mathematical Problems in Engineering
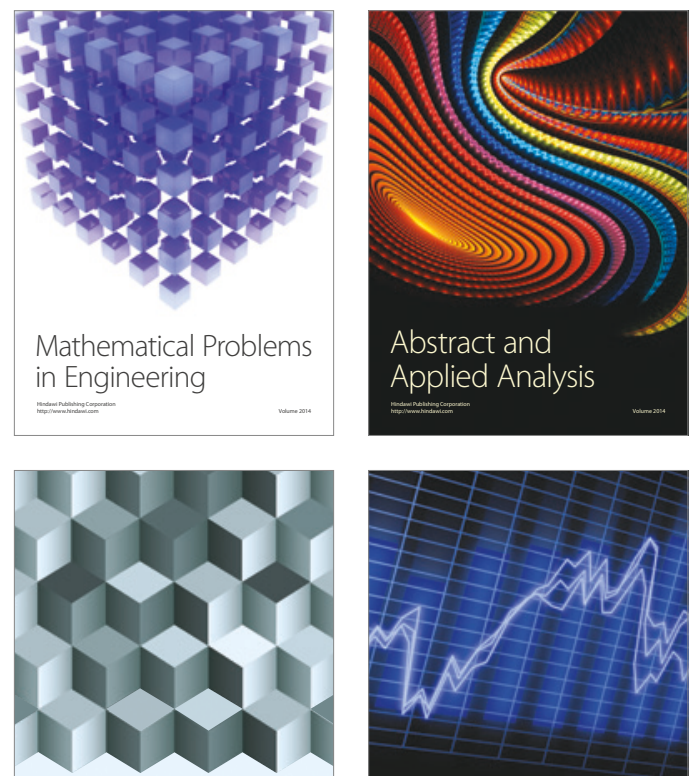

Journal of

Function Spaces

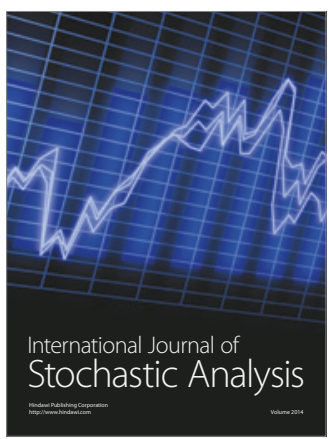

Probability and Statistics
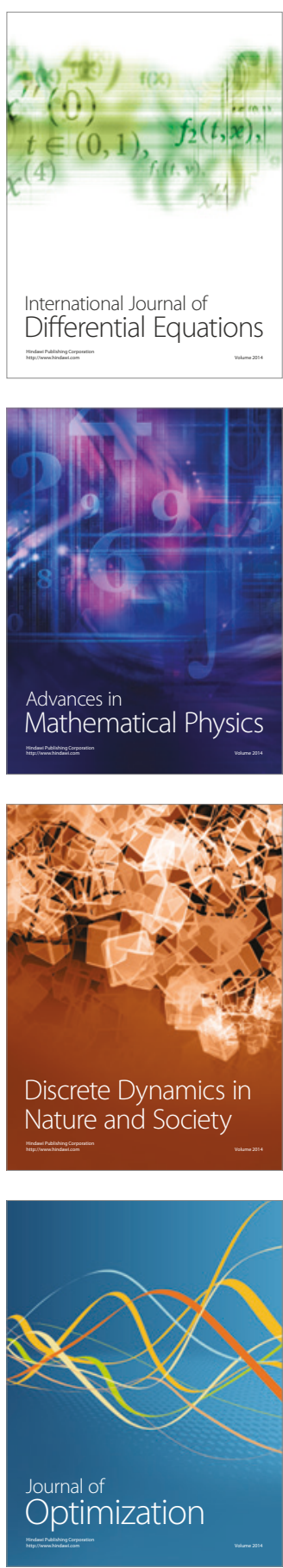